Review Article

\title{
Risk Factors for Mortality in Patients with Aortoesophageal Fistula Related to Aortic Lesions
}

\author{
Shan Li $\mathbb{D}^{1},{ }^{1}$ Feng Gao $\mathbb{D}^{1},{ }^{1}$ Hai-ou Hu $\mathbb{D},{ }^{2}$ Jin Shi $\mathbb{D},{ }^{1}$ and Jie Zhang $\mathbb{D}^{1}$ \\ ${ }^{1}$ Department of Gastroenterology, Beijing Anzhen Hospital, Capital Medical University, Beijing, China \\ ${ }^{2}$ Beijing Anzhen Hospital, Capital Medical University, No. 5A, Cardiosurgical Ward, Beijing, China \\ Correspondence should be addressed to Jie Zhang; zhangjie4155@sina.com
}

Received 12 May 2020; Revised 29 August 2020; Accepted 2 September 2020; Published 17 September 2020

Academic Editor: Jose Celso Ardengh

Copyright (C) 2020 Shan Li et al. This is an open access article distributed under the Creative Commons Attribution License, which permits unrestricted use, distribution, and reproduction in any medium, provided the original work is properly cited.

\begin{abstract}
Objective. Aortoesophageal fistula (AEF) related to aortic aneurysm and dissection is an uncommon but life-threatening condition. We performed a systematic review of risk factors for mortality and factors associated with the prognosis of AEF. Methods. A systematic search of the PubMed, Embase, and Cochrane Library databases was performed. Clinical characteristics, diagnostic methods, and treatments were assessed in terms of their ability to predict mortality. Results. The systematic review identified 184 eligible articles including 219 patients with AEF. Multivariable Cox regression revealed positive correlations of hemorrhagic shock (hazard ratio (HR): 1.824, 95\% CI: 1.217-2.735, $P=0.004$ ), sepsis (HR: 1.714, 95\% CI: 1.112-2.641, $P=0.015$ ), multiorgan failure (HR: 3.060, 95\% CI: 1.470-6.368, $P=0.003$ ), and conservative treatment (HR: 5.257, 95\% CI: 3.405-8.116, $P<0.001$ ) with mortality and a negative correlation between combination therapy (aortic graft replacement and esophagectomy) and mortality (HR: 0.319, 95\% CI: 0.125-0.813, $P=0.017$ ). Kaplan-Meier survival analysis showed that the 1-year cumulative survival rate was $42.5 \pm 3.8 \%$. The overall fistula-related mortality rate was $47.0 \%(103 / 219)$. The most common causes of death were bleeding (54.9\%) and infection (29.2\%). Conclusions. We found that hemorrhagic shock, sepsis, and multiorgan failure were risk factors for death in patients with AEF. Additionally, conservative treatment was associated with a higher rate of mortality, while combined aortic graft replacement and esophagectomy improved the prognosis.
\end{abstract}

\section{Introduction}

Aortoesophageal fistula (AEF) usually results in fatal gastrointestinal bleeding and is associated with a high mortality rate, despite the use of advanced surgical and endovascular techniques [1]. Previous studies reported that nearly $60 \%$ of patients with AEF died within 6 months; the 1 -year survival rate was only $28 \%[2,3]$. Since the 1990s, thoracic endovascular aortic repair (TEVAR) has been increasingly used as a less invasive treatment for aortic aneurysm and aortic dissection [4]; however, postTEVAR AEF has been frequently observed as a fatal complication [5]. Due to the rarity of AEF, few studies have investigated its risk factors for mortality, or the longterm prognosis [2]. Therefore, we systematically reviewed the literature on AEF related to aortic lesions, including aortic aneurysm and dissection. We reviewed clinical char- acteristics, diagnostic methods, treatment options, and prognosis of AEF, especially regarding factors associated with poor outcomes of AEF.

\section{Methods}

2.1. Search Strategy. The review was performed following the Preferred Reporting Items for Systematic Reviews and MetaAnalysis (PRISMA) guidelines. A systematic search was undertaken in the PubMed, Embase, and Cochrane Library databases from 2000 to March 6, 2020. The search terms were "aortic aneurysm," "aneurysm, dissecting," "endovascular procedures," "aortic surgery," "endograft," "stent graft," and "esophageal fistula." Only articles written in English were included. The references of retrieved articles were reviewed manually to identify missed studies. 
2.2. Eligibility Criteria. Studies were included in accordance with the following eligibility criteria: (1) observational studies, case reports, or case series; (2) sufficient information for analysis including outcomes and management strategies; (3) AEF associated only with aortic aneurysm or dissection; and (4) full-text article or meeting abstract. Duplicate studies were excluded; only the latest version was included in the analysis when the information was inconsistent.

2.3. Data Extraction. All articles were independently reviewed, and data were extracted by two reviewers (SL and FG); the final decision regarding inclusion was made by another reviewer (JZ). The following data were analyzed: age and sex; location and size of aortic aneurysm or dissection; a history of aortic surgery and/or endovascular treatment and the interval between the latest procedure and the occurrence of AEF; comorbidity and clinical symptoms (chest/abdominal/back pain, dysphagia, fever, and hemorrhage); elevated inflammatory markers and positive bacterial culture; diagnostic methods and related manifestations; location and size of fistula; treatments; and follow-up duration and prognosis.

2.4. Statistical Analysis. Categorical variables are described as percentages and continuous variables as means \pm standard deviations or medians. Comparisons of the primary and secondary AEF groups were performed by the Wilcoxon twosample test, Fisher's exact test, or $\chi^{2}$ test, as appropriate. The survival rate was evaluated by Kaplan-Meier analysis. Univariable and multivariable Cox regression analyses were conducted to identify factors independently related to the outcomes of interest. We excluded variables from the univariable analysis for multivariable regression model if their differences were not significant between groups and if the percentage of missing data was more than $15 \%$ of all the AEF patients. A $P$ value of $<0.05$ was considered indicative of statistical significance. Statistical analysis was performed using SPSS ver. 21.0 software (IBM ${ }^{\circledR}$, Armonk, NY).

\section{Results}

The systematic review identified 184 eligible articles that included 219 patients with AEF. The mean patient age was $65.8 \pm 13.5$ years, and $63.1 \%$ of patients were men. Detailed information on the included articles is provided in the supplementary file (available here). The PRISMA flowchart is shown in Figure 1.

3.1. Demographic and Clinical Features. The baseline characteristics of the survivors were similar to those of the nonsurvivors, except for age and hemorrhage shock (Table 1). Compared with the nonsurvivors, the survivors were significantly younger $(62.4 \pm 13.2$ vs. $68.9 \pm 13.0$ years, $P<0.001)$ and less presented frequent hemorrhage shock $(37.1 \%$ vs. $62.2 \%, P<0.001)$. There were no differences in fistula location, radiographic findings, or endoscopic findings between the two groups.

In our study, $7.8 \%(17 / 219)$ of total AEF patients were diagnosed as mycotic aortic aneurysms (AA). 47.1\% (8/17) of which were primary AEFs, and 52.9\% (9/17) were second- ary AEFs which means AEF were confirmed after mycotic AA treated by graft replacement or TEVAR. For mycotic AA, the proportion of primary AEFs was significantly higher in survivors than in nonsurvivors while secondary AEFs took a larger proportion in nonsurvivors ( $85.7 \%$ vs.20.0\%, $14.3 \%$ vs. $80.0 \%, P=0.015$, Table 2 ). The mortality rate of patients with AEF after primary mycotic AA treated with TEVAR was extremely high $(100 \%, 5 / 5)$, but the difference was not statistically significant due to the small sample size $(P=0.444)$. Positive cultures were mentioned in 61 cases, and the top three prevalent agents were Staphylococcus aureus $(36.1 \%, 22 / 61)$, Streptococcus species $(21.3 \%, 13 / 61)$, and Candida species $(21.3 \%, 13 / 61)$.

3.2. Treatment. The treatments for aortic and esophageal lesions are shown in Table 3. For aortic lesions, 32.9\% of the patients underwent graft replacement, $21.5 \%$ underwent TEVAR, and $8.7 \%$ underwent both graft replacement and TEVAR. Only the graft replacement rate differed between survivors and nonsurvivors $(49.5 \%$ vs. $18.1 \%, P<0.001)$. Both Dacron graft and cryopreserved aortic allograft were common choices and differed between survivors and nonsurvivors $(43.0 \%$ vs. $14.0 \%, P<0.001 ; 15.0 \%$ vs. $6.1 \%, P=0.042$, Table 4). Among the 76 patients who received no treatment for aortic lesions, 10 survived, and 66 died (9.7\% vs. 56.9\%, $P<0.001)$. Regarding esophageal lesions, $27.9 \%$ of the patients underwent esophagectomy, $16.0 \%$ underwent fistula repair, $10.0 \%$ received esophageal stents, and $5.0 \%$ received combination esophageal treatments. Only esophagectomy was performed more frequently in survivors than in nonsurvivors (45.6\% vs. $12.1 \%, P<0.001$ ). Among the 90 patients who received no esophageal treatments, 18 survived, and 72 died $(17.5 \%$ vs. $62.1 \%, P<0.001)$. The therapies used in patients with AEF are summarized in Table 2. The combination of aortic graft replacement and esophagectomy was the only therapy performed more frequently in survivors than in nonsurvivors $(30.1 \%$ vs. $6.0 \%, P<0.001)$. In total, 55 of 57 patients who received no treatment for aortic or esophageal lesions had a fatal outcome (mortality rate of 94.5\%). Furthermore, $47.8 \%$ of patients with a follow-up period longer than 4 weeks received prolonged antibiotic treatment $(>4$ weeks); however, the difference was not statistically significant between the groups (53.3\% vs. $37.0 \%, P=0.102)$.

3.3. Short-Term Outcomes. The overall in-hospital mortality rate was $42.0 \%(94 / 219)$. Complications were observed in 84 patients $(38.4 \%$, Table 1$)$; this rate was significantly lower in survivors than in nonsurvivors $(28.2 \%$ vs. $47.4 \%, P=0.004$ ). Sepsis $(23.3 \%)$ was the most common complication, followed by pulmonary complications (11.4\%) and fistula recurrence $(6.8 \%)$. Only sepsis and multiorgan failure rates significantly differed between survivors and nonsurvivors (10.7\% vs. $34.5 \%, P<0.001$; 0 vs. $9.5 \%, P=0.001$ ). Six patients $(2.7 \%)$ underwent reintervention within 30 days; the rate did not differ between the two groups.

3.4. Long-Term Outcomes. The mean follow-up period was 8.6 months (interquartile range: 7 days-10.9 months). Kaplan-Meier survival analysis showed that the 1-year 


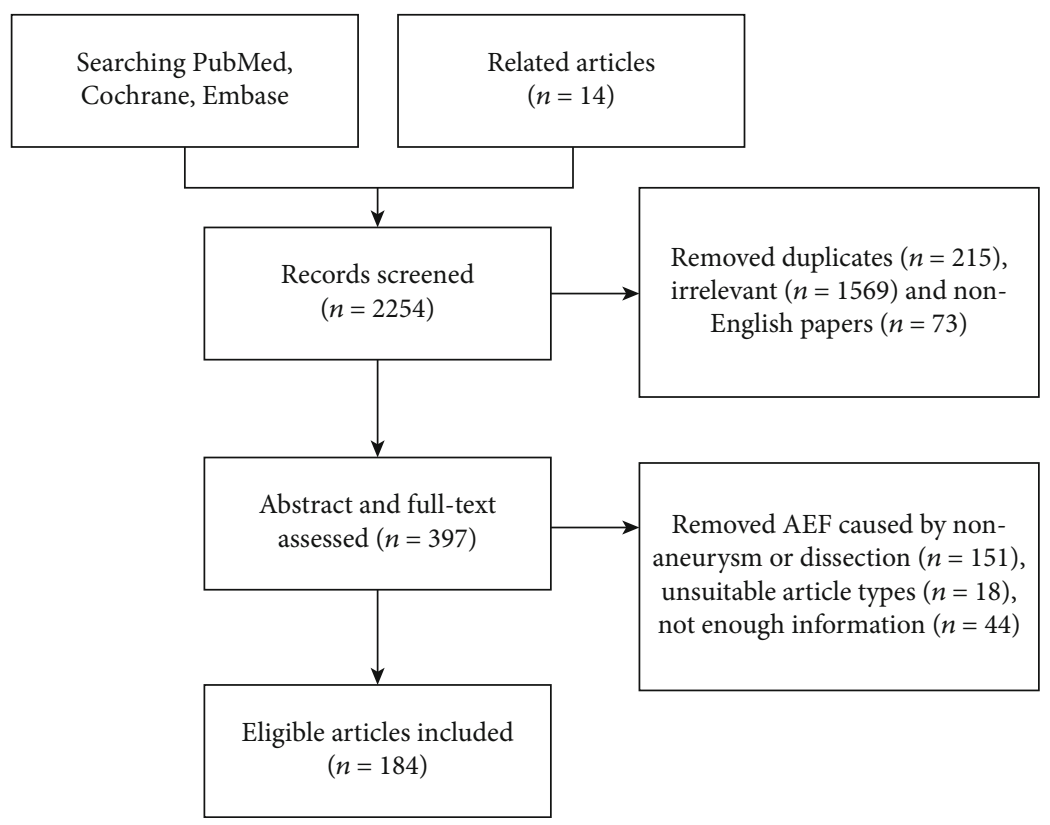

Figure 1: PRISMA flowchart.

cumulative survival rate was $42.5 \pm 3.8 \%$. The overall fistularelated mortality rate was $47.0 \%(103 / 219)$. The most common causes of death were bleeding $(54.9 \%)$ and infection (29.2\%). Fourteen patients (6.4\%) underwent unplanned reintervention after 30 days; the rate did not differ between the two groups.

The overall survival rate of patients with AEF who underwent surgery for aortic or esophageal lesions was significantly higher than that of nonsurgical patients $(P<0.001$, Figure 2$)$. Concerning aortic therapy, a better prognosis was observed in patients treated with graft replacement than in patients treated with TEVAR $(P=0.047)$; however, no difference was found between the graft replacement plus TEVAR combination treatment and any single treatment (both $P>0.05$ ). The worst prognosis was found among patients who received no treatment for aortic lesions $(P<0.001)$. Regarding esophageal therapy, patients who underwent esophagectomy had better long-term survival compared to patients who underwent esophageal repair, esophageal stent, or no treatment $(P=0.040, P=0.001$, and $P<0.001)$. No difference in the survival rate was found between patients who underwent esophageal repair and those who underwent esophageal stent $(P=0.276)$; both of these groups had better survival rates than patients who received no treatment $(P<0.001$, $P=0.024)$. Better long-term survival was observed in patients who underwent aortic graft replacement or esophagectomy compared to those who received other surgeries or treatments $(P=0.043, P<0.001)$.

3.5. Prognostic Factors. Univariable regression analysis showed that the odds of death increased with age and aortic rupture $(P=0.001, P=0.012$, Table 5) and decreased with the graft replacement plus TEVAR combination treatment $(P=0.042)$; however, these factors were not significant in a multivariable regression analysis $(P=0.587$,
$P=0.064, P=0.189)$. Contrast leak in CT and aneurysm size were also associated with mortality $(P=0.018, P=0.005)$ but were not included in the multivariable Cox regression analysis due to the lack of information in more than $15 \%$ of patients with AEF. Significant positive correlations of hemorrhagic shock, sepsis, multiorgan failure, and conservative treatment with mortality were found in multivariable regression analysis $(P=0.004, P=0.015, P=0.003$, and $P<0.001)$, while aortic graft replacement and esophagectomy combination treatment was associated with significantly lower mortality $(P=0.017)$.

\section{Discussion}

AEF is a rare cause of upper gastrointestinal bleeding classically characterized by Chiari's triad [6], consisting of chest pain and sentinel hemorrhage followed by fatal exsanguination after an asymptomatic interval. In our study, hemorrhagic shock was the only initial presentation that was more frequently observed in patients with fatal AEF $(P<0.001)$; moreover, it was associated with mortality in multivariable analysis $(P=0.004)$. These findings were consistent with the results of prior studies, which also reported that hemodynamic shock was a risk factor for in-hospital mortality $[7,8]$. Other studies reported that ectopic gas in the mediastina, a typical computed tomography finding of AEF [9], was associated with mortality; however, this was not confirmed in our study. And we found contrast leak in CT was associated with mortality in univariable analyses $(P=0.018)$. Aneurysm size also differed between survivors and nonsurvivors $(P=0.015)$ and was significantly associated with mortality in univariable Cox analysis $(P=0.005)$. Large thoracic aneurysms directly compress the esophagus and cause dysphagia, which may lead to aortoesophageal or aortobronchial fistulization [10-12]. Czerny et al. reported 
TABLE 1: Demographic, clinical, radiographic, and endoscopic findings of patients with AEF.

\begin{tabular}{|c|c|c|c|c|}
\hline & Total $n=219$ & Survivors $n=103$ & Nonsurvivors $n=116$ & $P$ \\
\hline Male sex & $63.1 \%(137 / 217)$ & $62.7 \%(64 / 102)$ & $63.5 \%(73 / 115)$ & 1.000 \\
\hline Mean age (years) & $65.8 \pm 13.5$ & $62.4 \pm 13.2$ & $68.9 \pm 13.0$ & $<0.001$ \\
\hline Type of AEF & & & & 0.077 \\
\hline Primary & $44.7 \%(98 / 219)$ & $51.5 \%(53 / 103)$ & $38.8 \%(45 / 116)$ & \\
\hline Secondary & $55.3 \%(121 / 219)$ & $48.5 \%(50 / 103)$ & $61.2 \%(71 / 116)$ & \\
\hline \multicolumn{5}{|l|}{ Comorbidity } \\
\hline Hypertension & $34.5 \%(48 / 139)$ & $28.8 \%(19 / 66)$ & $39.7 \%(29 / 73)$ & 0.212 \\
\hline Other arterial diseases & $24.5 \%(34 / 139)$ & $21.2 \%(14 / 66)$ & $27.4 \%(20 / 73)$ & 0.434 \\
\hline Coronary heart disease & $13.7 \%(19 / 139)$ & $16.7 \%(11 / 66)$ & $11.0 \%(8 / 73)$ & 0.459 \\
\hline COPD & $12.9 \%(18 / 139)$ & $12.1 \%(8 / 66)$ & $13.7 \%(10 / 73)$ & 0.806 \\
\hline Renal disease & $12.2 \%(17 / 139)$ & $9.1 \%(6 / 66)$ & $15.1 \%(11 / 73)$ & 0.312 \\
\hline Diabetes & $9.4 \%(13 / 139)$ & $4.5 \%(3 / 66)$ & $13.7 \%(10 / 73)$ & 0.082 \\
\hline Hyperlipidemia & $7.2 \%(10 / 139)$ & $3.0 \%(2 / 66)$ & $11.0 \%(8 / 73)$ & 0.101 \\
\hline \multicolumn{5}{|l|}{ Clinical presentation } \\
\hline Hemorrhage & $86.3 \%(183 / 212)$ & $86.0 \%(86 / 100)$ & $86.6 \%(97 / 112)$ & 1.000 \\
\hline Hemorrhagic shock & $50.5 \%(105 / 208)$ & $37.1 \%(36 / 97)$ & $62.2 \%(69 / 111)$ & $<0.001$ \\
\hline Chest/abdominal/back pain & $38.3 \%(79 / 206)$ & $39.6 \%(38 / 96)$ & $37.3 \%(41 / 110)$ & 0.775 \\
\hline Dysphagia & $11.7 \%(24 / 206)$ & $12.5 \%(12 / 96)$ & $10.9 \%(12 / 110)$ & 0.829 \\
\hline Fever & $25.2 \%(52 / 206)$ & $27.1 \%(26 / 96)$ & $23.6 \%(26 / 110)$ & 0.631 \\
\hline Chiari's triad & $20.0 \%(41 / 205)$ & $15.8 \%(15 / 95)$ & $23.6 \%(26 / 110)$ & 0.220 \\
\hline Elevated inflammatory markers & $73.3 \%(63 / 86)$ & $77.1 \%(27 / 35)$ & $70.6 \%(36 / 51)$ & 0.622 \\
\hline Positive cultures & $27.9 \%(61 / 219)$ & $26.2 \%(27 / 103)$ & $29.3 \%(34 / 116)$ & 0.652 \\
\hline Mycotic AA & $7.8 \%(17 / 219)$ & $6.8 \%(7 / 103)$ & $8.6 \%(10 / 116)$ & 0.801 \\
\hline \multicolumn{5}{|l|}{ Imaging features } \\
\hline Mediastinal air bubbles in CT & $39.9 \%(71 / 178)$ & $35.2 \%(32 / 91)$ & $44.8 \%(39 / 87)$ & 0.221 \\
\hline Contrast leak in CT & $11.2 \%(20 / 178)$ & $8.8 \%(8 / 91)$ & $13.8 \%(12 / 87)$ & 0.346 \\
\hline Contrast leak in angiography & $36.7 \%(11 / 30)$ & $44.4 \%(8 / 18)$ & $25.0 \%(3 / 12)$ & 0.442 \\
\hline Contrast leak in esophagogram & $73.7 \%(14 / 19)$ & $60.0 \%(6 / 10)$ & $88.9 \%(8 / 9)$ & 0.303 \\
\hline \multicolumn{5}{|l|}{ Endoscopic features } \\
\hline Tumor-like submucosal mass & $21.9 \%(35 / 160)$ & $22.1 \%(17 / 77)$ & $21.7 \%(18 / 83)$ & 1.000 \\
\hline Blood clot & $40.0 \%(64 / 160)$ & $41.6 \%(32 / 77)$ & $38.6 \%(32 / 83)$ & 0.748 \\
\hline Mucosa necrosis & $42.5 \%(68 / 160)$ & $45.5 \%(35 / 77)$ & $39.8 \%(33 / 83)$ & 0.523 \\
\hline Pulsatile arterial bleeding & $7.5 \%(12 / 160)$ & $7.8 \%(6 / 77)$ & $7.2 \%(6 / 83)$ & 1.000 \\
\hline Visible aortic wall or graft & $23.1 \%(37 / 160)$ & $22.1 \%(17 / 77)$ & $24.1 \%(20 / 83)$ & 0.852 \\
\hline Type of aortic lesion & & & & 1.000 \\
\hline Aneurysm & $84.5 \%(185 / 219)$ & $84.5 \%(87 / 103)$ & $84.5 \%(98 / 116)$ & \\
\hline Dissection & $15.5 \%(34 / 219)$ & $15.5 \%(16 / 103)$ & $15.5 \%(18 / 116)$ & \\
\hline \multicolumn{5}{|l|}{ Location of fistula } \\
\hline Descending aorta & $89.0 \%(195 / 219)$ & $90.3 \%(93 / 103)$ & $87.9 \%(102 / 116)$ & 0.667 \\
\hline Aortic arch & $17.4 \%(38 / 219)$ & $15.5 \%(16 / 103)$ & $19.0 \%(22 / 116)$ & 0.593 \\
\hline Ascending aorta & $2.7 \%(6 / 219)$ & $3.9 \%(4 / 103)$ & $1.7 \%(2 / 116)$ & 0.424 \\
\hline Upper esophagus & $19.4 \%(24 / 124)$ & $18.6 \%(11 / 59)$ & $20.0 \%(13 / 65)$ & 0.743 \\
\hline Middle esophagus & $66.1 \%(82 / 124)$ & $62.7 \%(37 / 59)$ & $69.2 \%(45 / 65)$ & 0.455 \\
\hline Lower esophagus & $16.1 \%(20 / 124)$ & $18.6 \%(11 / 59)$ & $13.8 \%(9 / 65)$ & 0.626 \\
\hline Fistula size $(\mathrm{cm})$ & $2.2 \pm 1.8(60 / 219)$ & $2.0 \pm 1.6(30 / 60)$ & $2.4 \pm 2.0(30 / 60)$ & 0.391 \\
\hline Aneurysm size $(\mathrm{cm})$ & $6.7 \pm 3.0(72 / 219)$ & $5.8 \pm 2.6(33 / 72)$ & $7.5 \pm 3.2(39 / 72)$ & 0.015 \\
\hline Complications & $38.4 \%(84 / 219)$ & $28.2 \%(29 / 103)$ & $47.4 \%(55 / 116)$ & 0.004 \\
\hline Sepsis & $23.3 \%(51 / 219)$ & $10.7 \%(11 / 103)$ & $34.5 \%(40 / 116)$ & $<0.001$ \\
\hline
\end{tabular}


TABLe 1: Continued.

\begin{tabular}{|c|c|c|c|c|}
\hline & Total $n=219$ & Survivors $n=103$ & Nonsurvivors $n=116$ & $P$ \\
\hline Multiorgan failure & $5.0 \%(11 / 219)$ & 0 & $9.5 \%(11 / 116)$ & 0.001 \\
\hline Pulmonary complications & $11.4 \%(25 / 219)$ & $13.6 \%(14 / 103)$ & $9.5 \%(11 / 116)$ & 0.397 \\
\hline Renal failure & $3.2 \%(7 / 219)$ & $1.9 \%(2 / 103)$ & $4.3 \%(5 / 116)$ & 0.451 \\
\hline Neural complications & $4.1 \%(9 / 219)$ & $2.9 \%(3 / 103)$ & $5.2 \%(6 / 116)$ & 0.506 \\
\hline Aortic rupture & $2.3 \%(5 / 219)$ & 0 & $4.3 \%(5 / 116)$ & 0.062 \\
\hline Fistula recurrence & $6.8 \%(15 / 219)$ & $6.8 \%(7 / 103)$ & $6.9 \%(8 / 116)$ & 1.000 \\
\hline Re-interventions within $30 \mathrm{~d}$ & $2.7 \%(6 / 219)$ & $2.9 \%(3 / 103)$ & $2.6 \%(3 / 116)$ & 1.000 \\
\hline Reinterventions unplanned after $30 \mathrm{~d}$ & $6.4 \%(14 / 219)$ & $7.8 \%(8 / 103)$ & $5.2 \%(6 / 116)$ & 0.582 \\
\hline
\end{tabular}

AA: aortic aneurysm; AEF: aortoesophageal fistula; CT: computed tomography; SD: standard deviation.

Data are counts, percentages, means \pm standard deviations or medians. Numbers of patients with available data are shown in parentheses.

TABLE 2: Survival data for mycotic AA in AEF patients.

\begin{tabular}{llll}
\hline & Total $n=17$ & Survivors $n=7$ & Nonsurvivors $n=10$ \\
\hline $\begin{array}{l}\text { Type of AEFs } \\
\text { Primary AEFs }\end{array}$ & $47.1 \%(8 / 17)$ & & 0.015 \\
Secondary AEFs & $52.9 \%(9 / 17)$ & $14.3 \%(1 / 7)$ & $20.0 \%(2 / 10)$ \\
\hline
\end{tabular}

AA: aortic aneurysm; AEF: aortoesophageal fistula.

that aneurysm size was related to the development of postTEVAR AEF due to mechanical compression and secondary erosion [3, 13]; esophageal ischemia and mediastinal infection were cited as potential causes. Notably, there was no significant difference in aneurysm size between patients with primary and secondary AEF.

Although mycotic AA accounts for $7.8 \%$ of total AEF patients, positive blood or tissue cultures were reported in up to $27.9 \%$ of AEF patients, dominated by Staphylococcus aureus, Streptococcus species, and Candida species. No significant differences were found in AEF types (primary or secondary AEF) between survivors and nonsurvivors; but for mycotic AA, primary AEF took a larger proportion in survivors while secondary AEF was just the opposite $(P=0.015)$. These findings emphasized the importance of broadspectrum antibiotic or even antifungal therapy, especially for secondary AEF.

The overall in-hospital mortality rate was $42.0 \%$; complications were observed in $38.4 \%$ of patients with AEF. Sepsis was the most common complication and was significantly correlated with mortality rate $(P=0.015)$, which emphasizes the role of infection control in the treatment of AEF [14]. Canaud et al. reported that use of broad-spectrum antibiotics for longer than 4 weeks in patients with AEF reduced the mortality rate [15]; in our study, $47.8 \%$ of patients with a follow-up period longer than 4 weeks received prolonged antibiotic treatment, but no significant difference was found between survivors and nonsurvivors $(P=0.102)$. Considering the risk of infection, broad-spectrum antibiotics should be recommended for a certain period; however, lifelong oral administration remains controversial $[16,17]$. Other conservative treatments include proton pump inhibitors and jejunal nutrition [18]. Patients receiving such therapy are typically unable to undergo other treatments (especially surgery) due to their poor general condition and multiple comorbidities [19], and the outcome is almost invariably fatal for patients with $\operatorname{AEF}(55 / 57,96.5 \%)$.

Surgery for AEF is performed to repair the fistula and control bleeding and infection $[20,21]$. The approaches include extra-anatomical bypass or in situ replacement with grafts, as well as repair or resection of esophageal fistula with primary or secondary reconstruction $[1,22,23]$. In our study, the overall survival rate of patients with AEF who underwent surgery either for aortic or esophageal lesions was significantly higher compared to that of nonsurgical patients with AEF $(P<0.001)$. Moulakakis et al. reported that surgical treatment improved the prognosis of patients with infected thoracic aortic endografts with aortic fistulas [24]. Furthermore, aortic graft replacement and esophagectomy combination therapy achieved a better prognosis than other surgeries $(P=0.043)$, in association with a significantly lower mortality rate $(P=0.017)$. The 1 -year survival rate reached $79.0 \%$, compared to $54.0 \%$ for other surgeries. A recent questionnaire survey also demonstrated that aortic replacement combined with esophagectomy improved the survival rate of post-TEVAR patients [25]. Other researchers advocated this strategy as the only radical treatment for AEF that achieved high success rates in patients with post-TEVAR AEF [26, 27]. These results indicated the importance of addressing sources of bleeding and infection [28]. However, the mortality rate of this approach reached $18.4 \%$ in our study and was associated with considerable complications that required attention [29]. Both Dacron graft and cryopreserved aortic allograft were common choices, and cryopreserved aortic allograft was considered to be resistant against infection with a relative low reinfection rate when treated with aortic infections [30]. But its availability, whether match the patients' aortic shape, and delay of transportation all might limit its 
TABLe 3: Treatments and outcomes of patients with AEF.

\begin{tabular}{|c|c|c|c|c|}
\hline & Total $n=219$ & Survivors $n=103$ & Nonsurvivors $n=116$ & $P$ \\
\hline \multicolumn{5}{|l|}{ Treatment for aortic lesions } \\
\hline Aortic repair & $2.3 \%(5 / 219)$ & $1.9 \%(2 / 103)$ & $2.6 \%(3 / 116)$ & 1.000 \\
\hline Aortic graft & $32.9 \%(72 / 219)$ & $49.5 \%(51 / 103)$ & $18.1 \%(21 / 116)$ & $<0.001$ \\
\hline TEVAR & $21.5 \%(47 / 219)$ & $26.2 \%(27 / 103)$ & $17.2 \%(20 / 116)$ & 0.138 \\
\hline Aortic graft+TEVAR & $8.7 \%(19 / 219)$ & $12.6 \%(13 / 103)$ & $5.2 \%(6 / 116)$ & 0.057 \\
\hline No treatment & $34.7 \%(76 / 219)$ & $9.7 \%(10 / 103)$ & $56.9 \%(66 / 116)$ & $<0.001$ \\
\hline \multicolumn{5}{|l|}{ Treatment for esophageal lesions } \\
\hline Esophageal repair & $16.0 \%(35 / 219)$ & $19.4 \%(20 / 103)$ & $12.9 \%(15 / 116)$ & 0.202 \\
\hline Esophagectomy & $27.9 \%(61 / 219)$ & $45.6 \%(47 / 103)$ & $12.1 \%(14 / 116)$ & $<0.001$ \\
\hline Esophageal stents & $10.0 \%(22 / 219)$ & $9.7 \%(10 / 103)$ & $10.3 \%(12 / 116)$ & 1.000 \\
\hline Esophageal combination treatments & $5.0 \%(11 / 219)$ & $7.8 \%(8 / 103)$ & $2.6 \%(3 / 116)$ & 0.120 \\
\hline No treatment & $41.1 \%(90 / 219)$ & $17.5 \%(18 / 103)$ & $62.1 \%(72 / 116)$ & $<0.001$ \\
\hline \multicolumn{5}{|l|}{ Combination of therapy } \\
\hline Conservative treatment & $26.0 \%(57 / 219)$ & $1.9 \%(2 / 103)$ & $47.4 \%(55 / 116)$ & $<0.001$ \\
\hline Aortic repair \pm esophageal surgery & $2.3 \%(5 / 219)$ & $1.9 \%(2 / 103)$ & $2.6 \%(3 / 116)$ & 1.000 \\
\hline TEVAR & $11.9 \%(26 / 219)$ & $12.6 \%(13 / 103)$ & $11.2 \%(13 / 116)$ & 0.835 \\
\hline TEVAR+esophageal repair & $2.3 \%(5 / 219)$ & $2.9 \%(3 / 103)$ & $1.7 \%(2 / 116)$ & 0.668 \\
\hline TEVAR+esophagectomy & $1.8 \%(4 / 219)$ & $2.9 \%(3 / 103)$ & $0.9 \%(1 / 116)$ & 0.344 \\
\hline TEVAR+esophageal stent & $4.6 \%(10 / 219)$ & $6.8 \%(7 / 103)$ & $2.6 \%(3 / 116)$ & 0.196 \\
\hline Aortic graft & $2.3 \%(5 / 219)$ & $1.9 \%(2 / 103)$ & $2.6 \%(3 / 116)$ & 1.000 \\
\hline Aortic graft+esophageal repair & $10.0 \%(22 / 219)$ & $12.6 \%(13 / 103)$ & $7.8 \%(9 / 116)$ & 0.265 \\
\hline Aortic graft+esophagectomy & $17.4 \%(38 / 219)$ & $30.1 \%(31 / 103)$ & $6.0 \%(7 / 116)$ & $<0.001$ \\
\hline Aortic graft+esophageal stent & $0.9 \%(2 / 219)$ & $1.0 \%(1 / 103)$ & $0.9 \%(1 / 116)$ & 1.000 \\
\hline TEVAR+aortic graft \pm esophageal treatments & $8.7 \%(19 / 219)$ & $12.6 \%(13 / 103)$ & $5.2 \%(6 / 116)$ & 0.057 \\
\hline TEVAR/aortic graft+esophageal treatments & $3.2 \%(7 / 219)$ & $4.9 \%(5 / 103)$ & $1.7 \%(2 / 116)$ & 0.258 \\
\hline Esophageal repair & $1.8 \%(4 / 219)$ & $1.0 \%(1 / 103)$ & $2.6 \%(3 / 116)$ & 0.624 \\
\hline Esophagectomy & $3.2 \%(7 / 219)$ & $5.8 \%(6 / 103)$ & $0.9 \%(1 / 116)$ & 0.053 \\
\hline Esophageal stent & $3.7 \%(8 / 219)$ & $1.0 \%(1 / 103)$ & $6.0 \%(7 / 116)$ & 0.069 \\
\hline Antibiotics (>4 weeks) & $47.8 \%(65 / 136)$ & $53.3 \%(48 / 90)$ & $37.0 \%(17 / 46)$ & 0.102 \\
\hline
\end{tabular}

AEF: aortoesophageal fistula; TEVAR: thoracic endovascular aortic repair.

Data are percentages. Numbers of patients with available data are shown in parentheses.

TABLE 4: Summary of grafts used for aortic replacement.

\begin{tabular}{|c|c|c|c|c|}
\hline & Total & Survivors & Nonsurvivors & $P$ \\
\hline Prosthetic grafts & $27.6 \%(59 / 214)$ & $43.0 \%(43 / 100)$ & $14.0 \%(16 / 114)$ & $<0.001$ \\
\hline PTFE grafts & $1.0 \%(2 / 201)$ & $2.2 \%(2 / 92)$ & 0 & 0.208 \\
\hline Dacron grafts & $23.9 \%(48 / 201)$ & $39.1 \%(36 / 92)$ & $11.0 \%(12 / 109)$ & $<0.001$ \\
\hline Cryopreserved aortic allograft & $10.3 \%(22 / 214)$ & $15.0 \%(15 / 100)$ & $6.1 \%(7 / 114)$ & 0.042 \\
\hline Pericardial tube & $0.5 \%(1 / 214)$ & $1.0 \%(1 / 100)$ & 0 & 0.467 \\
\hline
\end{tabular}

PTFE: polytetrafluoroethylene.

application in emergency surgery [31]. So antibiotic-soaked prosthetic grafts covered with omental flaps would be an effective alternative, especially when most of the AEF required emergency operations. Esophageal repair of the fistula was another option, especially when esophageal defects were minimal without gross contamination [32, 33].

TEVAR for patients with AEF has the advantages of rapid control of bleeding and minimal invasiveness [34], especially for patients with severe comorbidities [35, 36]. Although a better prognosis was observed in patients with AEF treated with TEVAR compared to those treated with conservative therapy $(P<0.001)$, no correlation was found between TEVAR and mortality in Cox regression $(P>0.05$ ). TEVAR can be recommended only as an emergency bridging therapy before surgery [37, 38]. Radical surgery should be performed as soon as possible once the 

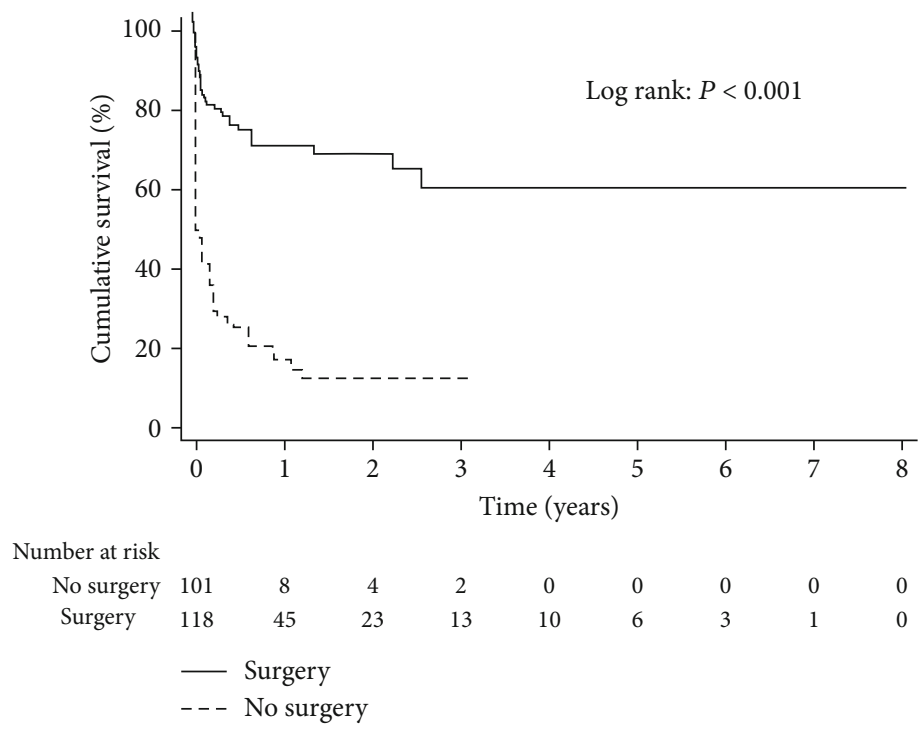

(a)

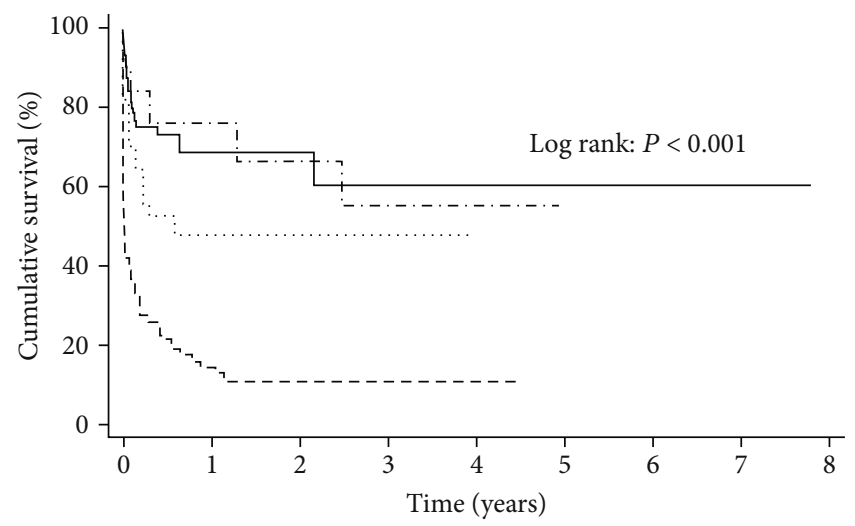

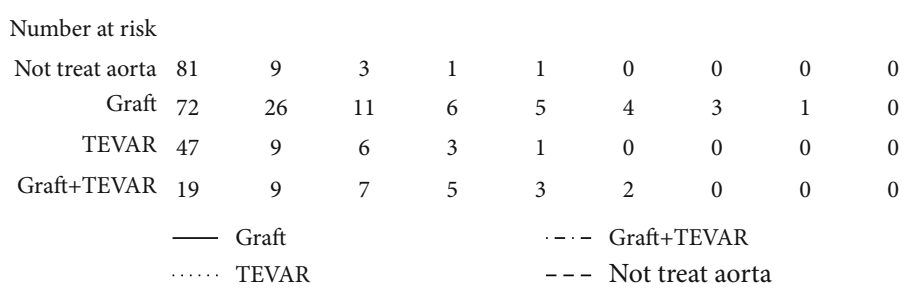

(b)

FIgURe 2: Continued. 

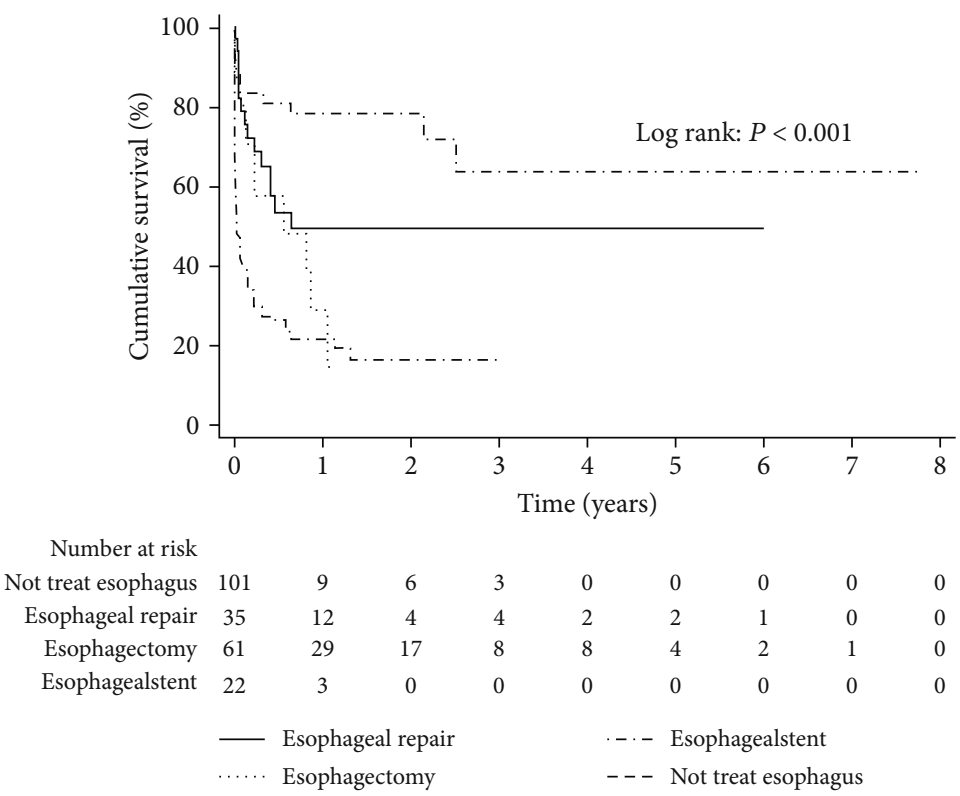

(c)
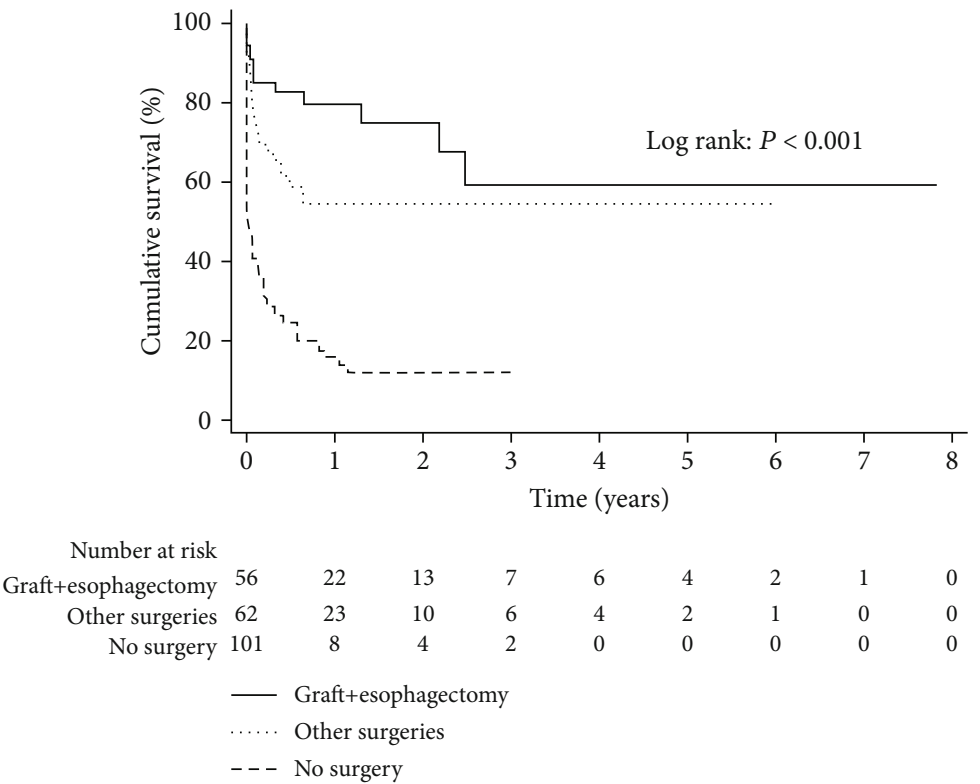

(d)

FIgURE 2: Overall survival of patients with aortoesophageal fistula (AEF). (a) Patients with AEF who underwent surgery for aortic or esophageal lesions had a significantly better prognosis compared to nonsurgical patients $(P<0.001)$. (b) Patients who were underwent graft replacement or graft replacement combined with TEVAR had a better prognosis than patients who received no treatment for aortic lesions (both $P<0.001$ ). (c) Patients who underwent esophagectomy had better long-term survival compared to patients who underwent esophageal repair, esophageal stent, or no treatment $(P=0.040, P=0.001$, and $P<0.001$, respectively). (d) Survival analysis confirmed better long-term survival of patients who underwent aortic graft replacement or esophagectomy compared to those who received other surgeries or treatments $(P=0.043, P<0.001)$.

condition is stable because TEVAR can control bleeding but cannot debride infection $[39,40]$. Sattah et al. recommended TEVAR as first-line treatment before aortic surgery for all patients with AEF, even those with stable hemodynamics [41]. However, we found that additional TEVAR with graft replacement was not superior to graft replacement alone $(P>0.05)$.
Endoscopic self-expanding esophageal stents are typically used for the palliation of malignant esophageal stricture; they can be helpful to control bleeding in esophageal fistula and prevent the entry of esophageal contents into the fistula [42]. An esophageal stent alone or combined with TEVAR could be a palliative option for patients who are unable to tolerate radical surgery $[43,44]$. Although the longest reported 
TABLE 5: Cox regression analyses.

\begin{tabular}{|c|c|c|c|c|c|c|}
\hline & \multicolumn{3}{|c|}{ Univariable Cox regression } & \multicolumn{3}{|c|}{ Multivariable Cox regression } \\
\hline & HR & $95 \% \mathrm{CI}$ & $P$ & HR & $95 \% \mathrm{CI}$ & $P$ \\
\hline$\overline{\text { Age }}$ & 1.027 & $1.011-1.043$ & 0.001 & 1.004 & $0.988-1.021$ & 0.587 \\
\hline Hemorrhagic shock & 2.124 & $1.444-3.123$ & $<0.001$ & 1.824 & $1.217-2.735$ & 0.004 \\
\hline Contrast leak in $\mathrm{CT} \dagger$ & 2.102 & $1.134-3.897$ & 0.018 & & & \\
\hline Aneurysm size $\dagger$ & 1.155 & $1.044-1.279$ & 0.005 & & & \\
\hline Sepsis & 1.900 & $1.291-2.795$ & 0.001 & 1.714 & $1.112-2.641$ & 0.015 \\
\hline Multiorgan failure & 3.544 & $1.872-6.710$ & $<0.001$ & 3.060 & $1.470-6.368$ & 0.003 \\
\hline Aortic rupture & 3.186 & $1.289-7.786$ & 0.012 & 2.410 & $0.949-6.117$ & 0.064 \\
\hline Conservative treatment & 6.570 & $4.459-9.681$ & $<0.001$ & 5.257 & $3.405-8.116$ & $<0.001$ \\
\hline Aortic graft+esophagectomy & 0.214 & $0.099-0.460$ & $<0.001$ & 0.319 & $0.125-0.813$ & 0.017 \\
\hline TEVAR+aortic graft \pm esophageal treatments & 0.424 & $0.186-0.968$ & 0.042 & 0.493 & $0.172-1.415$ & 0.189 \\
\hline
\end{tabular}

AEF: aortoesophageal fistula; TEVAR: thoracic endovascular aortic repair; CT: computed tomography; HR: hazard ratio; CI: confidence interval. $\dagger$ Contrast leak in CT and aneurysm size were excluded from multivariate regression analysis due to the lack of information in more than $15 \%$ of patients with AEF.

poststent survival has been 8 months [42], the prognosis is typically unfavorable [18].

This study had several limitations. First, the data were obtained from case reports and case series published by different researchers, and the lack of uniformity of reporting items could have affected the results. Second, publication bias might have existed because physicians are inclined to publish positive results and successful patient outcomes. A randomized prospective study was impossible because of the low incidence of AEF, so our findings can only be used for informational purposes.

\section{Conclusions}

In this study, we identified the risk factors for death in patients with AEF related to aortic aneurysm and dissection. Hemorrhagic shock, sepsis, and multiorgan failure were risk factors for death in patients with AEF. Additionally, conservative treatment was associated with a higher mortality rate, while combined aortic graft replacement and esophagectomy improved the prognosis. We hope that these findings can improve the prognosis of patients with AEF.

\section{Conflicts of Interest}

The authors declare no conflict of interest.

\section{Authors' Contributions}

Shan Li and Feng Gao systematically searched the databases and reviewed and extracted data independently; Shan Li analyzed data and contributed to drafting of the manuscript; Haiou $\mathrm{Hu}$ and Jin Shi critically revised the manuscript; and Jie Zhang contributed to the concept and design of the study.

\section{Acknowledgments}

The authors thank Dr. Yong-chen Hao, Department of Epidemiology, Beijing Anzhen Hospital, Capital Medical Uni- versity, Beijing Institute of Heart, Lung and Blood Vessel Diseases, for instruction in statistical analysis.

\section{Supplementary Materials}

Detailed information on the included articles is provided in the supplementary file. (Supplementary Materials)

\section{References}

[1] Y. Yang, D. Hu, and D. Peng, "Primary aortoesophageal fistula: a fatal outcome," The American Journal of Emergency Medicine, vol. 36, no. 2, p. 343.e1-3, 2018.

[2] K. Uno, T. Koike, S. Takahashi, D. Komazawa, and T. Shimosegawa, "Management of aorto-esophageal fistula secondary after thoracic endovascular aortic repair: a review of literature," Clinical Journal of Gastroenterology, vol. 10, no. 5, pp. 393-402, 2017.

[3] M. Czerny, H. Eggebrecht, G. Sodeck et al., "New insights regarding the incidence, presentation and treatment options of aorto-oesophageal fistulation after thoracic endovascular aortic repair: the European registry of endovascular aortic repair complications," European Journal of Cardio-Thoracic Surgery, vol. 45, no. 3, pp. 452-457, 2014.

[4] A. Kamigaichi, Y. Hamai, M. Emi et al., "Three-step surgical treatment of aortoesophageal fistula after thoracic endovascular aortic repair: a case report," International Journal of Surgery Case Reports, vol. 65, pp. 221-224, 2019.

[5] E. Stanger, A. M. Johnson, and J. Juneja Mucci, "Novel management of an aortoesophageal fistula prior to definitive surgical repair," Clinical Medicine Insights: Case Reports, vol. 12, p. 117954761983971, 2019.

[6] F. J. B. Slieker, J. P. Ruurda, and C. E. V. B. Hazenberg, "Esophageal resection, gastric tube reconstruction, and omental flap coverage of iatrogenic aortoesophageal fistula after secondary thoracic stentgraft infection: a case report," Annals of Vascular Surgery, vol. 59, pp. 314.e1-314.e4, 2019.

[7] V. X. Mosquera, M. Marini, F. Pombo-Felipe et al., "Predictors of outcome and different management of aortobronchial and aortoesophageal fistulas," The Journal of Thoracic and Cardiovascular Surgery, vol. 148, no. 6, pp. 3020-3026.e2, 2014. 
[8] F. H. Jonker, F. J. Schlösser, F. L. Moll et al., "Outcomes of thoracic endovascular aortic repair for aortobronchial and aortoesophageal fistulas," Journal of Endovascular Therapy, vol. 16, no. 4, pp. 428-440, 2009.

[9] A. Sipe, S. R. McWilliams, L. Saling, C. Raptis, V. Mellnick, and S. Bhalla, "The red connection: a review of aortic and arterial fistulae with an emphasis on CT findings," Emergency Radiology, vol. 24, no. 1, pp. 73-80, 2017.

[10] S. Mori, S. Kimura, A. Ro et al., "Two autopsy cases of rupture of the aorta by fistula formation after thoracic endovascular aortic repair and open stent-grafting on aortic arch aneurysm," Cardiovascular Pathology, vol. 39, pp. 61-66, 2019.

[11] M. Czerny, "Predictable or fateful-a short guide for how to anticipate risk to keep the incidence of aortoesophageal fistulation to a minimum," The Journal of Thoracic and Cardiovascular Surgery, vol. 155, no. 1, pp. 41-42, 2018.

[12] M. Luehr, C. D. Etz, M. Nozdrzykowski et al., "Emergency open surgery for aorto-oesophageal and aorto-bronchial fistulae after thoracic endovascular aortic repair: a single-centre experience," European Journal of Cardio-Thoracic Surgery, vol. 47, pp. 374-383, 2014.

[13] M. Yamamoto, H. Nishimori, M. Iguchi, and K. Orihashi, "Pathological investigation of graft-related oesophageal fistula," Interactive Cardiovascular and Thoracic Surgery, vol. 24, no. 5, pp. 813-814, 2017.

[14] T. Ito, T. Nobuoka, H. Sato et al., "Total surgical repair for secondary aortoesophageal fistula: two case reports," General Thoracic and Cardiovascular Surgery, vol. 68, no. 3, pp. 290294, 2020.

[15] L. Canaud, B. A. Ozdemir, W. W. Bee, S. Bahia, P. Holt, and M. Thompson, "Thoracic endovascular aortic repair in management of aortoesophageal fistulas," Journal of Vascular Surgery, vol. 59, no. 1, pp. 248-254, 2014.

[16] E. Qaja, M. Sivakumar, and M. Saleemi, “An unusual case of massive hematemesis caused by aorto-esophageal fistula due to mycotic aneurysm of mid-thoracic aorta in a patient without prior aortic instrumentation," Journal of Surgical Case Reports, vol. 2017, no. 5, 2017.

[17] S. Takeno, H. Ishii, A. Nanashima, and K. Nakamura, "Aortoesophageal fistula: review of trends in the last decade," Surgery Today, vol. 50, 2019.

[18] M. Sladojevic, M. Bjelovic, N. Ilic et al., "Open surgical treatment of secondary aortoesophageal and aortobronchial fistula after thoracic endovascular aortic repair and esophagocoloplasty in a second procedure," Annals of Vascular Surgery, vol. 44, pp. 417.e11-417.e16, 2017.

[19] T. Yamazato, T. Nakamura, N. Abe et al., "Surgical strategy for the treatment of aortoesophageal fistula," The Journal of Thoracic and Cardiovascular Surgery, vol. 155, no. 1, pp. 32-40, 2018.

[20] T. Nakamura, M. Yamamoto, T. Yamazato et al., "Surgical strategy of esophageal resection and reconstruction for aortoesophageal fistula," Diseases of the Esophagus, vol. 30, no. 9, pp. 1-7, 2017.

[21] A. M. Idhrees, A. Jacob, and B. V. Velayudhan, "An aortooesophageal fistula following endograft: sealing of fistulae with omentum and replacement of the aorta," Interactive Cardiovascular and Thoracic Surgery, vol. 26, no. 3, pp. 516-518, 2018.

[22] A. Omura, M. Yoshida, Y. Koda, and N. Mukohara, "Surgical management without resection of the oesophagus for aorto- oesophageal fistula secondary to aortic arch aneurysm rupture," Interactive Cardiovascular and Thoracic Surgery, vol. 23, no. 6, pp. 985-987, 2016.

[23] A. Majumder and R. B. Dharmaraj, "Successful endovascular management of a case of aorto-oesophageal fistula presenting as life threatening upper gastrointestinal bleed," EJVES Short Reports, vol. 39, pp. 29-32, 2018.

[24] K. G. Moulakakis, S. N. Mylonas, C. N. Antonopoulos et al., "Comparison of treatment strategies for thoracic endograft infection," Journal of Vascular Surgery, vol. 60, no. 4, pp. 1061-1071, 2014.

[25] M. Watanabe, M. Sato, M. Fukuchi, H. Kato, and H. Matsubara, "Treatment of aortoesophageal fistula developed after thoracic endovascular aortic repair: a questionnaire survey study," Esophagus, vol. 17, no. 1, pp. 81-86, 2020.

[26] J. H. Lee, B. Na, Y. Hwang, Y. H. Kim, I. K. Park, and K. H. Kim, "Surgical management of aorto-oesophageal fistula as a late complication after graft replacement for acute aortic dissection," Korean Journal of Thoracic and Cardiovascular Surgery, vol. 49, no. 1, pp. 54-58, 2016.

[27] M. S. Rawala, V. Badami, S. B. Rizvi, and A. Nanjundappa, "Aortoesophageal fistula: a fatal complication of thoracic endovascular aortic stent-graft placement," American Journal of Case Reports, vol. 19, pp. 1258-1261, 2018.

[28] K. Yamanaka, A. Omura, Y. Nomura et al., "Surgical strategy for aorta-related infection," European Journal of CardioThoracic Surgery, vol. 46, no. 6, pp. 974-980, 2014.

[29] C. I. Ochoa Chaar, M. A. Zafar, C. Velasquez, A. Saeyeldin, and J. A. Elefteriades, "Complex two-stage open surgical repair of an aortoesophageal fistula after thoracic endovascular aortic repair," Journal of Vascular Surgery Cases and Innovative Techniques, vol. 5, no. 3, pp. 261-263, 2019.

[30] K. Masabni, M. R. Weaver, P. Kandagatla et al., "Cryopreserved allograft in the management of native and prosthetic aortic infections," Annals of Vascular Surgery, vol. 56, pp. 110, 2019.

[31] M. R. Janko, S. Bose, and P. F. Lawrence, "Current status of treatment for aortic graft infection: when should cryopreserved allografts be used?," Seminars in Vascular Surgery, vol. 32, no. 1-2, pp. 81-87, 2019.

[32] S. H. Hwang, J. W. Cho, C. H. Bae, and J. S. Jang, "Staged surgical treatment of primary aortoesophageal fistula," The Korean Journal of Thoracic and Cardiovascular Surgery, vol. 52, no. 3, pp. 182-185, 2019.

[33] C. D. Hoang, "Esophageal injury: tissue is always the issue," The Journal of Thoracic and Cardiovascular Surgery, vol. 156, no. 1, article e27, p. e27, 2018.

[34] N. Takei, T. Kunieda, Y. Kumada, and M. Murayama, "Perigraft abscess subsequent to aortoesophageal fistula," Internal Medicine, vol. 57, no. 22, pp. 3255-3259, 2018.

[35] H. Akashi, S. Kawamoto, Y. Saiki et al., "Therapeutic strategy for treating aortoesophageal fistulas," General Thoracic and Cardiovascular Surgery, vol. 62, no. 10, pp. 573-580, 2014.

[36] M. Nozdrzykowski, J. Garbade, S. Leinung, A. Schmidt, F. W. Mohr, and M. A. Borger, "Thoracic endovascular aortic repair for aortoesophageal fistula after covered rupture of aortic homograft - a durable option?," AORTA, vol. 5, no. 3, pp. 96-100, 2017.

[37] S. Hakim, M. Gjeorgjievski, L. Garg, M. Orosey, and T. Desai, "Atypical aortoesophageal fistula with atypical and delayed presentation and negative imaging studies," Case Reports in 
Gastrointestinal Medicine, vol. 2016, Article ID 7219034, 5 pages, 2016.

[38] D. Kotelis, A. Gombert, and M. J. Jacobs, "Treatment of postthoracic endovascular aortic repair aorto-esophageal fistulaonly radical surgery can be effective: techniques and sequence of treatment," Journal of Thoracic Disease, vol. 10, no. 6, pp. 3869-3873, 2018.

[39] T. W. Lin and C. D. Kan, "Occult aortic fistulation affects late outcome of ruptured descending thoracic aortic aneurysms after emergency thoracic endovascular aortic repair in patients with initial hematemesis/hemoptysis," Formosan Journal of Surgery, vol. 51, no. 2, pp. 50-57, 2018.

[40] M. C. Firetto, M. Petrini, F. Sala, M. Domanin, G. Terribile, and P. R. Biondetti, "Thoracic aortic aneurysms, fistula, and thrombus," in Emergency Radiology of the Chest and Cardiovascular System. Medical Radiology, M. Scaglione, U. Linsenmaier, and G. Schueller, Eds., pp. 307-340, Springer, Cham, 2016.

[41] A. P. Sattah, M. H. Secrist, and S. Sarin, "Complications and perioperative management of patients undergoing thoracic endovascular aortic repair," Journal of Intensive Care Medicine, vol. 33, no. 7, pp. 394-406, 2016.

[42] U. Aday, D. A. Çetin, H. Çiyiltepe, E. Gündeş, E. Bozdağ, and A. S. Senger, "Cause of mortality in aortoesophageal fistula: oesophageal sepsis. A case report," Gastroenterology Review, vol. 3, no. 3, pp. 222-225, 2017.

[43] R. Spitaels, W. Jacob, F. Janssens et al., "Thoracic aortic aneurysm complicated by secondary aortoesophageal fistula after thoracic endovascular aortic repair: a case report," Acta Gastroenterologica Belgica, vol. 80, p. 527, 2017.

[44] M. Akin, T. Yalcinkaya, E. Alkan, G. Arslan, Y. Tuna, and B. Yildirim, "A cause of mortal massive upper gastrointestinal bleeding: aortoesophageal fistula," Medical Archives, vol. 70, no. 1, pp. 79-81, 2016. 